\title{
Conflict adaptation in time: Foreperiods as contextual cues for attentional adjustment
}

\author{
Mike Wendt • Andrea Kiesel
}

Published online: 23 June 2011

(C) Psychonomic Society, Inc. 2011

\begin{abstract}
Interference evoked by distractor stimulus information, such as flankers in the Eriksen task, is reduced when the proportion of conflicting stimuli is increased. This modulation is sensitive to contextual cues such as stimulus location or color, suggesting attentional adjustment to conflict contingencies on the basis of context information. In the present study, we explored whether conflict adjustment is modulated by temporal variation of conflict likelihood. To this end, we associated low and high proportions of conflict stimuli with foreperiods of different lengths. Flanker interference was higher with foreperiods associated with low conflict proportions, suggesting that participants use the foreperiod as a contextual cue for attentional adjustment. We conjecture that participants initially adopt the strategy useful for conflict contingencies associated with short foreperiods, and then readjust during the trial, in the absence of any additional exogenous cue, when the imperative stimulus has not occurred during a certain time interval.
\end{abstract}

Keywords Attention - Executive control Cognitive and attentional control

M. Wendt $(\bowtie)$

Experimental Psychology Unit, Helmut-Schmidt-University/

University of the Federal Armed Forces Hamburg,

Holstenhofweg 85,

22043 Hamburg, Germany

e-mail: mike.wendt@hsu-hh.de

\section{A. Kiesel}

Department of Psychology, University of Würzburg,

Röntgenring 11,

97070 Würzburg, Germany

e-mail: kiesel@psycholgie.uni-wuerzburg.de
Numerous experimental studies have demonstrated that performance in a given task is influenced by (formally) task-irrelevant stimulus information (i.e., stimulus information that is neither instructed nor needed for successful task performance), thereby demonstrating that processing is not confined to task-relevant information-or, in other words, that processing selectivity is incomplete. For instance, in the Eriksen flanker task (for an overview, see Eriksen, 1995), participants identify or categorize a target stimulus that is surrounded by irrelevant distractors (flankers). In this setting, participants usually respond more slowly and make more errors when the flankers are associated with a different response than the target (incongruent trials), as compared to conditions in which the flankers and target are associated with the same response (congruent trials). In the following, we denote the performance difference between congruent and incongruent trials as (flanker) interference.

It has been shown that the size of such interference depends on the proportions of congruent and incongruent trials. More precisely, interference decreases when the proportion of incongruent trials is increased and the proportion of congruent trials is reduced (e.g., Gratton, Coles, \& Donchin, 1992; Wendt \& Luna-Rodriguez, 2009). This proportion congruency effect has also been found in other conflict paradigms, such as the Stroop task (e.g., Tzelgov, Henik, \& Berger, 1992) and the Simon task (e.g., Hommel, 1994). Under the assumption that a smaller interference effect under perceptually identical conditions reflects a higher degree of processing selectivity, this modulation has been attributed to enhanced attentional focusing on the target stimulus dimension when processing of the distractor dimension is more likely to be detrimental to performance (e.g., Botvinick, Braver, Barch, Carter, \& Cohen, 2001; Gratton et al., 1992; Tzelgov et al., 1992). 
Findings from cuing procedures suggest that such attentional adjustment can occur within a trial, as demonstrated by smaller interference effects when an advance cue signals a high likelihood of the impending stimulus being incongruent rather than congruent (Fernandez-Duque \& Knight, 2008; Gratton et al., 1992). Recent studies have also shown that attentional adjustment can be based on implicit cues - that is, contextual stimulus features that correlate with the congruency level of the stimulus, even in cases in which the cue is presented simultaneously with the target and distractor stimuli. For instance, when stimuli randomly occur at varying locations that are associated with different congruent/incongruent proportions, smaller interference is observed at the location associated with a higher proportion of incongruent stimuli (Corballis \& Gratton, 2003; Crump, Gong, \& Milliken, 2006; Crump \& Milliken, 2009; Crump, Vaquero, \& Milliken, 2008; Vietze \& Wendt, 2009; Wendt, Kluwe, \& Vietze, 2008). Although results for contextual cues other than location, such as shape or color, have yielded mixed results (e.g., Crump et al., 2006; Crump et al., 2008; Heinemann, Kunde, \& Kiesel, 2009; Lehle \& Hübner, 2008; Vietze \& Wendt, 2009), it seems that, at least under some circumstances, attentional adjustment occurs "on the fly," based on encoding of the context feature.

Whereas most of the findings on context-dependent attentional adjustment can alternatively be accounted for in terms of practice-related associations involving specific distractors, responses, and context features, Crump and Milliken (2009) showed that this is not the whole story. Applying a Stroop task in which they varied the locationspecific proportion of incongruent trials for only a subset of the colors, these authors found that the location-specific proportion congruency effect generalized to another subset of colors that was presented with a constant congruent/ incongruent ratio at the two locations.

Yet so far, all studies demonstrating contextual attentional adjustment have made use of an (explicit or implicit) exogenous cue that signaled the likelihood of the upcoming congruency level. It has thus not been established whether context-specific conflict adjustments can be brought about by endogenous processes alone - that is, in the absence of an external triggering event. In the present study, we investigated this possibility by varying the likelihood of the upcoming congruency level during a time interval preceding the imperative stimulus. Specifically, we associated high and low congruent/incongruent proportions in an Eriksen flanker task with foreperiods of different lengths in the absence of any additional exogenous cue. Obtaining foreperiod-specific interference effects - that is, smaller interference for a foreperiod associated with a higher proportion of conflict stimuli-would indicate that participants use endogenous processes of time estimation as a contextual cue for attentional adjustment. Contrasting with all previous demonstrations of cue-based adjustment, which can be accounted for by assuming that adjustment is elicited by processing the contextual cue and either becomes effective instantaneously (in the case of simultaneous presentation of the cue and the imperative stimulus) or is maintained in a stereotype manner until onset of the imperative stimulus (in the case of precuing), foreperiodspecific adjustment would suggest optimized timing of the adjustment processes, thereby revealing a degree of flexibility thus far unknown.

Classical work on foreperiods has shown that the speed of responding to an imperative stimulus increases with foreperiod length if foreperiods of different lengths are presented with equal frequencies within the same block of experimental trials (e.g., Bertelson \& Tisseyre, 1968). This finding is consensually ascribed (a) to a positive relationship between response speed and some internal state of preparation and (b) to higher states of preparation at later moments of stimulus presentation. Concerning the effect of such nonspecific preparation on flanker interference, it is of interest that a recent study found larger interference effects when the foreperiod after which target and flanker stimuli occurred was expected due to valid cuing of that foreperiod, as compared to a condition of unexpected (i.e., invalidly cued) stimulus onset (Correa, Cappucci, Nobre, \& Lupiáñez, 2010). The authors attributed this finding to preparatory activation of all (i.e., both) responses, which might have increased the conflict evoked by an incongruent stimulus (for similar observations in the Simon task, see Fischer, Plessow, \& Kiesel, 2010). In conjunction with the above-noted assumption of an increased state of preparation after longer foreperiods, such a mechanism should yield larger interference effects for stimulus presentation after a long than after a short interval.

To control for such a general tendency (i.e., unrelated to congruency proportion) of interference-relevant processes to fluctuate over the course of the possible foreperiods in a trial, the experiment in the present study encompassed two conditions of foreperiod-congruency proportion contingency. Whereas for one group of participants conflict trials occurred predominantly after short foreperiods, for another group, conflict trials occurred predominantly after long foreperiods.

A potential problem for interpreting proportion congruency effects arises from the facts that modulations of interference have also been found as a function of the congruency level of the direct predecessor trial (i.e., lower interference after an incongruent than after a congruent trial; see, e.g., Gratton et al., 1992) and that trial-to-trial repetitions of the congruency level occur more frequently for a congruency level that is presented in a higher proportion. Although this sequential modulation of interference has also been accounted for in terms of attentional 
adjustment, ${ }^{1}$ an alternative interpretation relates to a confound between the sequence of congruency levels and the sequence of specific stimulus and response features on consecutive trials (Hommel, Proctor, \& Vu, 2004). More precisely, given a limited set of stimuli and responses, trials on which the congruency level alternates are associated with a higher proportion of repetition of either the target/ response or the distractor, combined with alternation of the other feature (so-called partial feature repetitions), than are trials on which the congruency level repeats. Assuming that partial feature repetitions constitute a processing disadvantage, due to a mismatch between the current stimulusresponse (S-R) demands and the retrieved memory episodes (referred to as event files; Hommel, 1998), a congruencylevel alternation cost - and thus a reduction of interference after an incongruent trial-is expected, even in the absence of any attentional adjustment.

Importantly, varying the likelihoods of congruent and incongruent trials in a foreperiod-specific manner implies that a congruency level, which is presented with increased frequency at one foreperiod, is preceded more frequently by a trial of the same congruency level associated with the same rather than with the alternative foreperiod. In contrast, the infrequent congruency level of a given foreperiod would be preceded more frequently by a trial of the other congruency level associated with the same rather than the alternative foreperiod. If foreperiod length acts as a retrieval cue for the eventfile of the previous trial or if foreperiod length is itself integrated in the event-file, this bias in trial-to-trial transitions should yield a benefit for the frequent congruency level and a cost for the infrequent congruency level of a given foreperiod, based on S-R feature repetition. To dismiss this possibility, we used a flanker task with four letters mapped onto four responses and confined the analyses to trials on which none of the stimulus or response features repeated from the directly preceding trial.

\section{Method}

\section{Participants}

The participants were 32 volunteers ( 23 women, 9 men) ranging in age from 19 to 32 years (mean 21.8), with

\footnotetext{
${ }^{1}$ In fact, the model of Botvinick, Braver, Barch, Carter, \& Cohen (2001) attributes both the sequential modulation and the congruencyproportion-dependent modulation of interference to the same mechanism. More precisely, in that model, the distribution of attentional weights across target and distractor stimulus dimensions is determined as a function of the congruency level of all preceding trials, weighted by their recency.
}

normal or corrected-to-normal vision. Participants took part in the experiment in partial fulfillment of a course requirement or in exchange for pay. Each participant attended a single experimental session lasting approximately 50-60 $\mathrm{min}$.

\section{Apparatus and stimuli}

An IBM-compatible computer equipped with a 17 -in. VGA display and the software package E-Prime (Schneider, Eschman, \& Zuccolotto, 2002) was used for stimulus presentation. A plus sign drawn in 36-point Courier New font served as the fixation cross. The stimuli were the letters $\mathrm{K}, \mathrm{L}, \mathrm{N}$, and $\mathrm{P}$, drawn in 32-point Arial font in white against a black background. The target letter was presented in the center of the screen and was surrounded by two flanking distractors on each side, the left and the righ (e.g., N N K N N). Space characters separated the letters from each other. Participants responded with the index and middle fingers of the right and left hands, and responses were collected using four external response keys. Two response keys were positioned next to each other, and the other two keys were positioned at a distance of about $25 \mathrm{~cm}$.

\section{Procedure}

A trial started with the presentation of a fixation cross, which was presented either for a short foreperiod of $200 \mathrm{~ms}$ or for a long foreperiod of $1,200 \mathrm{~ms}$ in the center of the screen. After the offset of the fixation cross, the target and distractors were displayed for $150 \mathrm{~ms}$. Responses were collected during a time window of $1,500 \mathrm{~ms}$ after target onset. Errors were indicated by the German word for error ("Fehler!"); in the case of missing responses, participants were requested to respond faster ("Bitte schneller!"). Both types of error feedback were accompanied by a tone signal. The next trial started $700 \mathrm{~ms}$ after response onset.

Participants were instructed to respond according to the centrally presented target letter and to ignore the flanking distractors. We used a Latin square to counterbalance the mapping of letters to the four response keys. At the end of each block, participants received feedback about their mean reaction time (RT) and number of errors in the block, and they were asked to try to respond faster without making more errors. Participants were not informed about the two different foreperiods or their relations to the frequency of flanker congruency.

Each participant performed in 10 experimental blocks of 120 trials each. In each experimental block, each of the four target letters was accompanied 15 times by one set of flanking distractors and 5 times by each of the other three flanking distractors, resulting in 60 congruent and 60 
incongruent trials. In half of the trials, the target and flankers appeared after a short foreperiod of $200 \mathrm{~ms}$, and in the other half of the trials, after a long foreperiod of $1,200 \mathrm{~ms}$. The proportions of congruent and incongruent trials varied for the two foreperiods. For half of the participants, there were 12 congruent and 48 incongruent target-flanker pairs after the short (200-ms) foreperiod and 48 congruent and 12 incongruent target-flanker pairs after the long (1,200-ms) foreperiod, and for the other half, the foreperiod-congruency proportion contingencies were reversed, resulting in congruency proportions per foreperiod of either $20 \%$ or $80 \%$ congruent trials. We refer to the former as the early conflict group and to the latter as the late conflict group.

\section{Results}

Only trials in which no letter was repeated from the previous trial were entered into the analysis. This led to the exclusion of $51.8 \%$ of the trials. Further, trials with RTs deviating more than 2.5 standard deviations from the mean RT of each experimental condition per participant (1.1\%) and trials that followed an error $(4.5 \%)$ were excluded from the analysis. For the remaining trials, mean RTs for correct trials and mean percentages of error (PEs) were computed for each participant depending on the within-subjects factors Congruency (congruent, incongruent) and Foreperiod $(200 \mathrm{~ms}, 1,200 \mathrm{~ms})$ and the between-subjects factor Congruency Proportion per Foreperiod (early conflict group, with $20 \%$ congruent and $80 \%$ congruent for the foreperiods $200 \mathrm{~ms}$ and $1,200 \mathrm{~ms}$, respectively, and late conflict group, with $80 \%$ congruent and $20 \%$ congruent for the foreperiods $200 \mathrm{~ms}$ and 1,200 ms, respectively). The averages of the mean RTs and mean error rates across participants are shown in Fig. 1.

\section{Reaction times}

An ANOVA with the between-subjects factor Congruency Proportion per Foreperiod and the within-subjects factors Congruency and Foreperiod revealed that participants responded faster in congruent $(604 \mathrm{~ms})$ than in incongruent $(647 \mathrm{~ms})$ trials, $F(1,30)=66.41, p<.001$. Further, the interaction of foreperiod and congruency approached significance, $F(1,30)=4.05, p=.053$, because the congruency effect was larger for the short foreperiod (49 $\mathrm{ms}$ ) than for the long foreperiod $(37 \mathrm{~ms})$. Most importantly, the factors Congruency Proportion per Foreperiod, Congruency, and Foreperiod interacted, $F(1,30)=$ $15.97, p<.001$. Participants of the late conflict group had a significantly larger congruency effect of $66 \mathrm{~ms}$ for the 200$\mathrm{ms}$ foreperiod, versus the congruency effect of $29 \mathrm{~ms}$ for the $1,200-\mathrm{ms}$ foreperiod, $t(15)=4.16, p<.001$. For the participants of the early conflict group, the congruency effect of $32 \mathrm{~ms}$ for the $200-\mathrm{ms}$ foreperiod was only numerically smaller than the congruency effect of $44 \mathrm{~ms}$ for the 1,200-ms foreperiod, $t(15)=1.44, p=.17 .^{2}$ No other effects were significant $(p>.34)$.

\section{Error rates}

The same ANOVA on error rates revealed that participants made fewer errors in congruent $(9.4 \%)$ than incongruent $(10.9 \%)$ trials, $F(1,30)=5.57, p=.025$. Further, participants made more errors for short $(10.8 \%)$ than for long $(8.7 \%)$ foreperiods, $F(1,30)=6.19, p=.019$. The interaction between foreperiod and congruency proportion per foreperiod was significant, $F(1,30)=7.11, p=.012$, reflecting the fact that participants of the late conflict group made more errors for the short $(12.1 \%)$ than for the long $(9.3 \%)$ foreperiods, whereas participants of the early conflict group made similar numbers of errors for both foreperiods $(9.5 \%$ and $9.6 \%$ for the foreperiods $200 \mathrm{~ms}$ and $1,200 \mathrm{~ms}$, respectively). The three-way interaction of the factors Congruency Proportion per Foreperiod, Congruency, and Foreperiod was not significant, $F(1,30)=0.0005, p=$ .994 . No other effects were significant $(p>.52)$.

\section{Discussion}

Interference from irrelevant stimulus dimensions when responding to a target stimulus shows that processing selectivity is often incomplete. Variations in the magnitude of such interference effects depending on the likelihoods of congruent and incongruent stimuli, however, suggest that the extents of processing relevant and irrelevant stimulus dimensions is, at least partly, under strategic control. Previous studies demonstrated a remarkable flexibility of attentional adjustment, showing that it is sensitive to contextual cues presented slightly before, or even simultaneously with, the target and distractor stimuli (e.g., Crump et al., 2006; Crump et al., 2008; Heinemann et al., 2009; Lehle \& Hübner, 2008; Vietze \& Wendt, 2009). Extending these findings, we investigated whether the length of a stimulus-free interval could act as contextual cue - that is,

\footnotetext{
${ }^{2}$ To investigate this asymmetry further, we increased power by collecting data from 16 new participants ( 8 for each of the two foreperiod-congruency contingency groups). Adding these data did not alter the previously observed data pattern. More precisely, the difference in congruency effects remained significant for the late conflict group (congruency effects amounting to $60 \mathrm{~ms}$ and $30 \mathrm{~ms}$ for the 200-ms foreperiod and the 1,200-ms foreperiod, respectively), and the difference between the congruency effects for the two foreperiods in the early conflict group remained nonsignificant (and was actually reduced to $4 \mathrm{~ms}$ ).
} 
Fig. 1 Mean reaction times and error rates as a function of foreperiod and congruency for the two conditions of proportion congruency per foreperiod. Error bars represent standard errors of the means

\section{late conflict group}

$80 \%$ - $20 \%$ congruent for $200 \mathrm{~ms}-1200 \mathrm{~ms}$
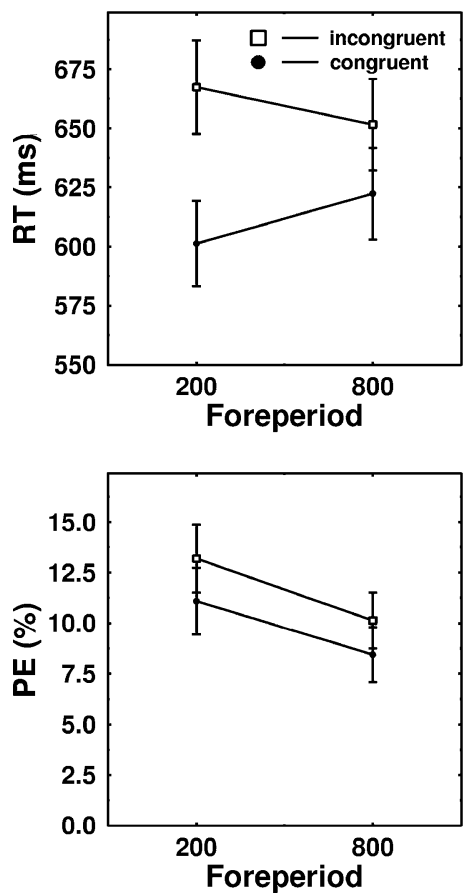

early conflict group

$20 \%$ - $80 \%$ congruent for $200 \mathrm{~ms}-1200 \mathrm{~ms}$
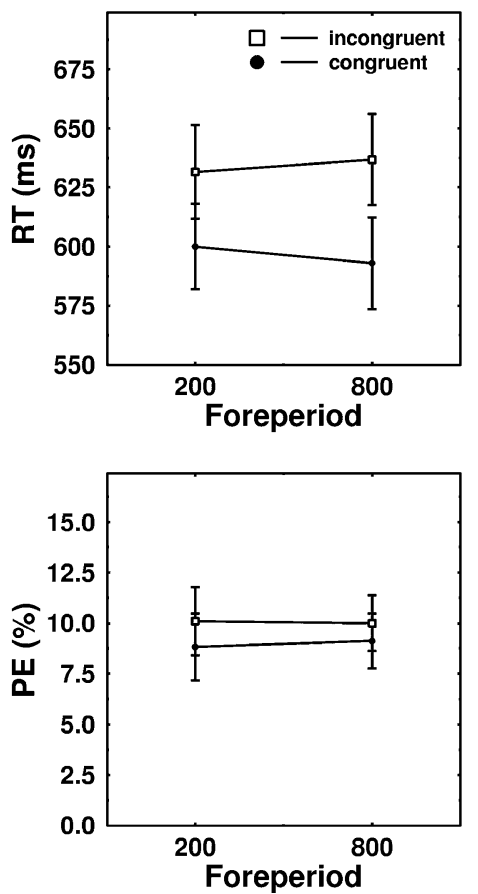

whether attentional adjustment according to to-be-expected conflict conditions would take place in the absence of any exogenous cue. In accordance with this assumption, the degree of flanker interference at short and long foreperiods was affected by the distribution of conflict proportions across the two foreperiods. Because we excluded all trials associated with any form of stimulus or response repetition from the data analysis, this foreperiod-specific proportion congruency effect cannot be accounted for in terms of a confound with partial S-R feature repetitions.

Noteworthily, foreperiod-dependent adjustment occurred despite the fact that we applied a task with four response alternatives. Recently, Wühr and Kunde (2008) demonstrated effective (explicit) cuing of the upcoming congruency level in a Simon task with two response alternatives, but not with three response alternatives. These authors assumed that participants tended to respond to the irrelevant location (applying an incompatible S-R mapping) if a cue indicated an incongruent trial. The adjustment effect obtained in our study cannot be accounted for by such distractor-response priming, because the flanker of an incongruent trial did not predict the required response. Rather, it seems that processing selectivity - that is, the distribution of attentional weights across target and distractor stimuli-was affected by the contingency of conflict proportion and foreperiod.

The fact that flanker interference for the two foreperiods differed only in the late conflict group suggests that the likelihood of a congruent or incongruent stimulus was not the only determinant of the development of the degree of processing selectivity after a warning signal. A possible reason for the group asymmetry was that conflict-dependent adjustment worked in conjunction with some general tendency to increase attentional focusing during the course of a preparation interval, thereby yielding differential interference effects regarding the two foreperiods when both factors worked in the same direction, and comparable effects when they worked in opposite directions. Although we can only speculate about the origin of such a tendency, it does not seem implausible to assume that participants are reluctant to abandon a highly focused state, which ensures correct performance, in the absence of an external event such as a congruent trial. The specific type of warning signal (i.e., the fixation cross) may have played a role here, possibly supporting fast summoning of spatial attention on the target position, at least in the late conflict group.

Although conflict adjustment in the flanker task has been accounted for in terms of spatial attention (e.g., Botvinick et al., 2001; Gratton et al., 1992), another, conflict-unrelated factor may have affected flanker interference by a different mechanism. Nonspecific response preparation, as envisaged by Correa, Cappucci, Nobre, \& Lupiáñez (2010), might be considered here. To account for the asymmetrical findings between the two experimental groups, one would have to assume, however, that such nonspecific response preparation decreased during the course of the (long) foreperiod, an interpretation that would run counter to the widely shared assumption that longer foreperiods are associated with a higher state of nonspecific preparation. 
Leaving aside additional factors influencing flanker interference, it seems straightforward to assume that participants began a trial by applying the degree of selectivity optimized for the congruency level likely to occur after the short foreperiod, and started to adjust their attentional set after a certain time interval in which no imperative stimulus was presented. An alternative possibility is that the length of the current foreperiod acted as a retrieval cue regarding the corresponding attentional set at the time of encoding of the imperative stimulus. In the latter case, the conflict adjustment observed in our study would resemble previous demonstrations of adjustment on the fly, albeit its triggering signal would be endogenously inferred instead of exogenously presented. ${ }^{3}$ The group asymmetry found seems, at least, consistent with the notion of adjustment taking place prior to stimulus presentation. Assuming that a higher degree of selectivity constitutes a less automatic state that benefits more strongly from increased preparation time, one might expect a significant reduction of interference effects to occur only with a long foreperiod. ${ }^{4}$ A reason for corresponding (i.e., foreperiodspecific) differences regarding retrieval of the attentional set seems less obvious.

Modulations of proportion congruency effects have recently been accounted for in terms of connectionist modeling. More precisely, in the model of Verguts and Notebaert (2008; see also Blais, Robidoux, Risko, \& Besner, 2007), the occurrence of conflict results in strengthening of the connection between a unit representing the currently present relevant stimulus feature (e.g., the color red on a Stroop task trial) and a task demand unit. The task demand unit feeds extra activation into the pathways responsible for the translation of the relevant stimulus dimension into required responses. As a consequence, more activation of a task demand unit facilitates S-R translation for this specific stimulus feature on future occasions associated with the same task, and thus accounts for findings of reduced interference for specific stimuli that occur more frequently in conflict conditions (e.g., Jacoby, Lindsay, \& Hessels, 2003). Although such models might be extended to account for context-dependent proportion congruency effects, it can be doubted whether dynamic

\footnotetext{
${ }^{3}$ These alternatives may be linked to the question of whether participants made use of the foreperiod cue on a voluntary basis. The present data do not allow us to decide on this issue, because we obtained no measure of awareness or conscious intention. Nevertheless, the fact that we did not tell participants about the foreperiodcongruency level contingencies demonstrates that foreperiod-specific adjustment can result as a consequence of incidental learning. This seems a noteworthy aspect of this study, if only because Crump, Vaquero, \& Milliken (2008) failed to obtain context-specific adjustment based on shape cues in a Stroop task, despite explicitly informing participants about the contingencies used.

${ }^{4}$ We thank an anonymous reviewer for pointing out this explanation.
}

within-trial adjustment, as suggested in the present study, is best explained by assuming trial-by-trial alterations of connection weights. An obvious theoretical alternative would be to assume that attentional weighting of perceptual dimensions is influenced by conflict expectation, which is continuously (or, at least, at times of probable stimulus onset) updated during the course of a foreperiod. Thus, the findings of the present study suggest that information from various sources, external and internal, is taken into consideration to optimize the attentional strategy for the specific to-be-expected conditions of an upcoming task.

\section{References}

Bertelson, P., \& Tisseyre, F. (1968). The time-course of preparation with regular and irregular foreperiods. Quarterly Journal of Experimental Psychology, 20, 297-300. doi:10.1080/ 14640746808400165

Blais, C., Robidoux, S., Risko, E. F., \& Besner, D. (2007). Itemspecific adaptation and the conflict-monitoring hypothesis: A computational model. Psychological Review, 114, 1076-1086.

Botvinick, M. M., Braver, T. S., Barch, D. M., Carter, C. S., \& Cohen, J. D. (2001). Conflict monitoring and cognitive control. Psychological Review, 108, 624-652.

Corballis, P. M., \& Gratton, G. (2003). Independent control of processing strategies for different locations in the visual field. Biological Psychology, 64, 191-209.

Correa, Á., Cappucci, P., Nobre, A. C., \& Lupiáñez, J. (2010). The two sides of temporal orienting: Facilitating perceptual selection, disrupting response selection. Experimental Psychology, 57, 142148. doi:10.1027/1618-3169/a000018

Crump, M. J. C., Gong, Z., \& Milliken, B. (2006). The contextspecific proportion congruent Stroop effect: Location as a contextual cue. Psychonomic Bulletin \& Review, 13, 316-321.

Crump, M. J. C., \& Milliken, B. (2009). The flexibility of contextspecific control: Evidence for context-driven generalization of item-specific control settings. Quarterly Journal of Experimental Psychology, 62, 1523-1532.

Crump, M. J. C., Vaquero, J. M. M., \& Milliken, B. (2008). Contextspecific learning and control: The roles of awareness, task relevance, and relative salience. Consciousness and Cognition, $17,22-36$

Eriksen, C. W. (1995). The flanker task and response competition: A useful tool for investigating a variety of cognitive problems. Visual Cognition, 2, 101-118.

Fernandez-Duque, D., \& Knight, M. B. (2008). Cognitive control: Dynamic, sustained, and voluntary influences. Journal of Experimental Psychology. Human Perception and Performance, 34, 340-355.

Fischer, R., Plessow, F., \& Kiesel, A. (2010). Auditory warning signals affect mechanisms of response selection: Evidence from a Simon task. Experimental Psychology, 57, 89-97.

Gratton, G., Coles, M. G. H., \& Donchin, E. (1992). Optimizing the use of information: Strategic control of activation of responses. Journal of Experimental Psychology. General, 121, 480-506.

Heinemann, A., Kunde, W., \& Kiesel, A. (2009). Context-specific prime-congruency effects: On the role of conscious stimulus representations for cognitive control. Consciousness and Cognition, 18, 966-976. 
Hommel, B. (1994). Spontaneous decay of response code activation. Psychological Research, 56, 261-268.

Hommel, B. (1998). Event files: Evidence for automatic integration of stimulus-response episodes. Visual Cognition, 5, 183-216. doi:10.1080/713756773

Hommel, B., Proctor, R. W., \& Vu, K.-P. L. (2004). A featureintegration account of sequential effects in the Simon task. Psychological Research, 68, 1-17.

Jacoby, L. L., Lindsay, D. S., \& Hessels, S. (2003). Item-specific control of automatic processes: Stroop process dissiociations. Psychonomic Bulletin \& Review, 10, 638-644.

Lehle, C., \& Hübner, R. (2008). On-the-fly adaptation of selectivity in the flanker task. Psychonomic Bulletin \& Review, 15, 814-818.

Schneider, W., Eschman, A., \& Zuccolotto, A. (2002). E-Prime user's guide. Pittsburgh: Psychology Software Tools.

Tzelgov, J., Henik, A., \& Berger, J. (1992). Controlling Stroop effects by manipulating expectations for color words. Memory \& Cognition, 20, 727-735. doi:10.3758/BF03202722
Verguts, T., \& Notebaert, W. (2008). Hebbian learning of cognitive control: Dealing with specific and nonspecific adaptation. Psychological Review, 115, 518-525.

Vietze, I., \& Wendt, M. (2009). Context-specificity of conflict frequency-dependent control. Quarterly Journal of Experimental Psychology, 62, 1391-1400.

Wendt, M., Kluwe, R. H., \& Vietze, I. (2008). Location-specific versus hemisphere-specific adaptation of processing selectivity. Psychonomic Bulletin \& Review, 15, 135-140. doi:10.3758/ PBR.15.1.135

Wendt, M., \& Luna-Rodriguez, A. (2009). Conflict-frequency affects flanker interference: Role of stimulus-ensemble-specific practice and flanker-response contingencies. Experimental Psychology, 56, 206-217. doi:10.1027/1618-3169.56.3.206

Wühr, P., \& Kunde, W. (2008). Pre-cueing spatial S-R correspondence: Is there regulation of expected response conflict? Journal of Experimental Psychology. Human Perception and Performance, 34, 872-883. 